\title{
A Human Aware Mobile Robot Motion Planner
}

\author{
Emrah Akin Sisbot, Luis F. Marin-Urias, Rachid Alami, and Thierry Siméon, Member, IEEE
}

\begin{abstract}
Robot navigation in the presence of humans raises new issues for motion planning and control when the humans must be taken explicitly into account. We claim that a humanaware motion planner must not only provide safe robot paths, but also synthesize good, socially acceptable and legible paths.

This paper focuses on a motion planner that takes explicitly into account its human partners by reasoning about their accessibility, their vision field and their preferences in terms of relative human-robot placement and motions in realistic environments. This planner is part of a human-aware motion and manipulation planning and control system that we aim to develop in order to achieve motion and manipulation tasks in the presence or in synergy with humans.
\end{abstract}

Index Terms-HRI, Motion Planning, Social Interaction

\section{INTRODUCTION}

$\mathbf{T}$ HE introduction of robots in our daily life raises a key issue that is "added" to the "standard challenge" of autonomous robots: the presence of humans in the robot environment and the necessity to interact with them. In the factory, the robot is systematically physically separated from the human workers. This will not be the case for future applications where the robot will be in situation where it will have to assist humans.

To allow the robots "co-exist" with humans, human-robot interaction needs to be taken into account in all steps of the robot design. This paper addresses issues related to the close interaction between humans and robots from the standpoint of the motion decisions that must be taken by the robot in order to ensure:

- Safe motion, i.e., that does not harm the human,

- Reliable and effective motion, i.e, that achieves the task adequately considering the motion capacities of the robot,

- Socially acceptable motion, i.e, that takes into account a motion model of the human as well as his preferences and needs.

Let us consider a simple "fetch and carry task" as illustrated in figure 1 for a socially interactive robot [1]. The robot has to perform motion and manipulation actions and should be able to determine where a given task should be achieved, how to place itself relatively to a human, how to approach him, how to hand the object and how to move in a relatively constrained environment in the presence of humans (an apartment for instance). Our goal is to develop a robot that is able to take into account "social constraints" and to synthesize plans compatible with human preferences, acceptable by humans and easily legible in terms of intention.

This work is part of a broader effort to develop a decisional framework for human-robot interactive task achievement, embedded in a cognitive architecture, aimed to allow the robot

Authors are with LAAS/CNRS, University of Toulouse, 7, avenue du Colonel Roche, 31077, Toulouse, France. Email: \{LastName@laas.fr $\}$ not only to accomplish its tasks but also to produce behaviours that support its commitment vis-a-vis its human partner and also to interpret human behaviours and intentions [2]

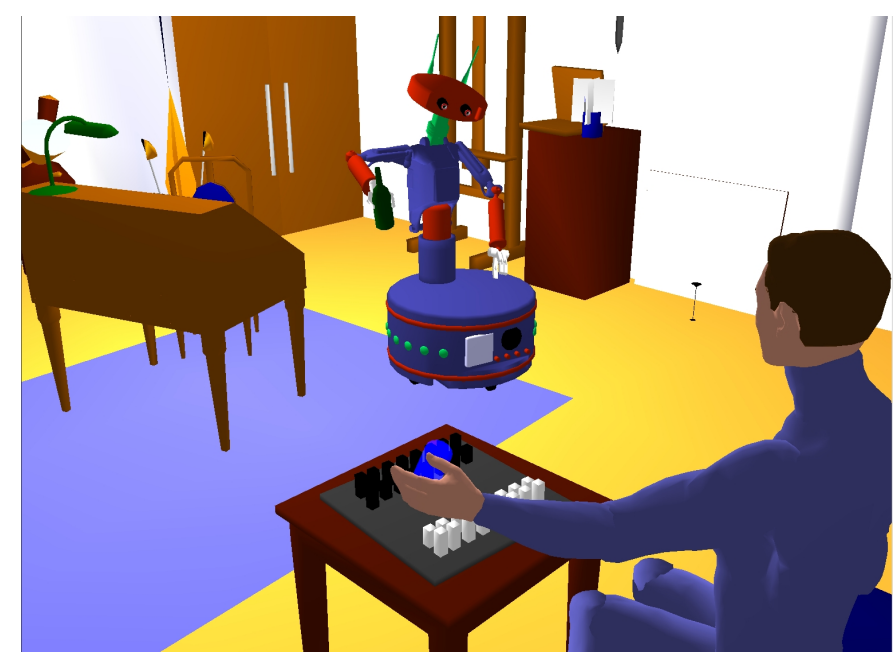

Fig. 1. A "fetch-and-carry" scenario in a domestic environment in presence of a person.

We have introduced our approach and presented preliminary results in [3], [4]. We have discussed in [5] how user studies have influenced the design of our planner. In this paper, we present in detail a Human Aware Motion Planner (HAMP) and its implementation with simulation and real world results.

In Section II, we briefly discuss related work. Section III provides the main characteristics and algorithms of our motion planner. We show simulation results in different scenarios in Section IV. Finally, we describe in Section V the implementation of the planner on a mobile robot and present real-world results.

\section{RELATED WORK}

Although human-robot interaction is a very active research field, there is not extensive research on motion planning in the presence of humans.

In the factory, safety is assured by not allowing humans to approach robots at work. Although this method mostly prevents collision risks, it cannot be applied in applications where the robot has to assist, sometimes physically, a human. Obviously safety issues become the primary concern when robots come into humans' everyday environment. The notion of safety becomes very critical and must be studied in detail with all of its aspects [6].

In user studies conducted by Nonaka et al. [7], two aspects of human's safety have been studied: "physical" safety and 
"mental" safety. With this separation, the notion of safety extends its meaning by including not only physical aspects but also psychological effects of the robot's motions on humans.

Physical safety is absolutely necessary for the humanrobot interaction. It must be assured during the hardware and software design process of the robot. Ikuta et al. classify safety strategies in two different categories: "design" strategies and "control" strategies. Besides new designs [8], [9] that will ensure safety at the physical level, fault-tolerant approaches [10] tend to detect and limit the consequences of hardware and software problems. A danger index is considered in control strategies and robot motions are executed by minimizing this index [11]-[13].

With these approaches physical safety is assured by avoiding collisions with humans and by minimizing the intensity of the impact in case of a collision. Another issue is illustrated by the research on smart wheelchairs. Although there is no direct interaction between the chair and the transported person, the wheelchair motion needs to take into account implicitly the human comfort [14].

In usual interactions between humans, some non-written rules are respected and determine the distance between two persons (see the proxemics theory of E. T. Hall [15]). The robot should comply to similar conventions [1] [16].

To achieve more human friendly behaviors, there is much work trying to imitate human motions and to better understand how humans behave in social environments. [17] describes a behavior-based method for placing the robot like a person in a multi-partnered conversation. In recent work by Pacchierotti et al. [18], [19], a human-robot hallway passage scenario is studied and "social patterns" for relative human-robot placement are extracted from these studies. These patterns are encoded into robot behaviors and result in a human friendly motions for a specific hallway crossing scenario.

Another approach that deals not only with safety but also implicitly with comfort issues is the work on velocity profiles along a planned trajectory by Krishna et al. [20]. Here the robot adapts its trajectory and its speed to optimize the execution time and also to guarantee that no collision will occur in a dynamic environment. Although the human is not considered explicitly, this method guarantees a motion without collision by taking into account the sensor capabilities of the robot as well as its dynamics and the dynamics of the environment. Since the sensors have a certain range, it is likely necessary to slow down in some places of the robot's trajectory where the sensor's field of view is blocked by narrow passages, doors or corners.

Although several authors propose motion planning or reactive schemes considering humans, there is no contribution that tackles globally the problem that we consider in this paper.

\section{Human Aware Navigation Planning}

User studies on robot motion and approach direction with respect to humans [21] [5] provided us a number of properties and non-written rules/protocols [15] of human-robot or human-human interactions. Only very limited works consider such properties and often in an ad hoc manner. We describe below a new technique that integrates such additional constraints in a more generic way. First, we introduce two additional criteria to the motion planning stage in order to ensure human safety and comfort. These criteria, namely "safety criterion" and "visibility criterion", present two important aspects of robot navigation in a human-robot interaction scenario.

Each criterion is represented by a set of numerical values stored in a 2D grid. This criterion grid contains a set of cells with various costs derived from the relative positions of humans in the environment, humans' states, their capabilities, and preferences. A criterion grid $G$ is defined as:

$$
G=\left(M_{n, p}, H_{1} \ldots H_{n}, f\right)
$$

where $M_{n, p}$ is a matrix containing $n * p$ cells represented by $a_{i, j}$, the cost of the coordinate $(i, j)$ in the grid, $H_{1} \ldots H_{n}$ is the list of humans in the environment. The function $f$ calculates the value of each cell according to its coordinate by taking into account only one human. The matrix $M$ is constructed by the equation:

$$
a_{i, j}=\max _{k}\left(f\left(H_{k}, i, j\right)\right)
$$

A human $H_{i}$ is modeled by $H_{i}=\left(\right.$ St, State $_{1} \ldots$ State $\left._{n}\right)$ where $S t$ is the structure and kinematics of the human and State $_{i}$ is a human state defined by a number of cost parameters. A state is defined by:

$$
\text { State }_{i}=(\text { Name }, \text { Conf }, \text { Param })
$$

where Name is the name of a posture state (e.g. Name = SITTING, STANDING), Conf is the human's configuration in that state (if applicable) and Param represents the data needed to compute costs according to that state.

We will further explain below the structure of the "safety" and of the "visibility" criteria and their underlying properties.

\section{A. Safety Criterion}

The first criterion, called "safety criterion", mainly focuses on ensuring the safety of the robot and the humans by controlling the distance between these two. This property aims to keep a distance between the robot and the humans in the environment. However in some cases, as in the necessity of a close interaction (e.g. handling an object), the robot has to approach the person whom it wants to interact with. Therefore, the distance between the robot and the human is neither uniform nor fixed and depends on the interaction. The feeling of safety is highly dependent on the human's personality, his physical capabilities and his actual states; for example, safety differs highly in a sitting position compared to standing. When the human is sitting, his mobility is reduced and he tends to have a low tolerance to the robot getting close. On the contrary, when standing up he has a higher mobility, thus allowing the robot to come closer.

These properties are treated in the current system by a "safety grid". This grid contains a human centered Gaussian form of cost distribution. Each coordinate $(x, y)$ in this grid contains a cost inversely proportional to the distance to the human. When the distance between the human and a point in the environment (in the grid) $D\left(x_{i}, y_{j}\right)$ is greater than the distance of another point $D\left(x_{k}, y_{l}\right)$, we have $\operatorname{Cost}\left(x_{k}, y_{l}\right)>$ 
$\operatorname{Cost}\left(x_{i}, y_{j}\right)$. Since the safety concerns lose their importance when the robot is far away from the human, the cost also decreases when getting farther from the human, until some maximal distance at which it becomes null.

Figure 2 shows a computed safety grid attached to a sitting/standing human. The height of the vertical lines represents the cost associated with each cell. As shown in the figure, human's current state (sitting, standing, etc) plays an important role in the cost of the grid. Also note that this approach allows us to consider other types of human states.

Once this grid is computed, searching for a minimum cost path will result in a motion that avoids moving too close to the human unless it is necessary. However, if the environment is constrained or if the task requires so, the robot is allowed to approach to the human. Only very close proximity of the human is strictly prohibited to avoid collisions.

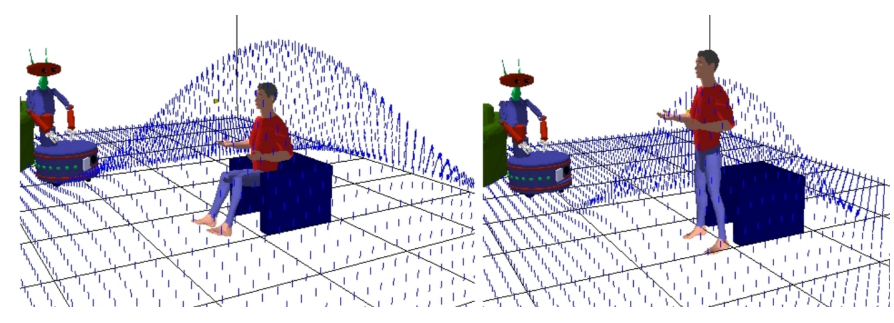

Fig. 2. A Safety grid is built around every human in the environment. It depends highly on the human's posture. As the person feels less "threatened" when standing, the value and the range of the costs are less important.

\section{B. Visibility Criterion}

The second criterion, called "visibility criterion", aims to improve human comfort during robot's motion. Humans generally feel more comfortable when the robot is in their field of view. This criterion allows the robot to be mostly in the human's field of view during its motions.

The resulting grid, namely "visibility grid", is constructed according to costs reflecting the effort required by the human to get the robot in his field of view. For example, grid points located in a direction for which the human only has to move his eyes have a lower cost than positions requiring him to move his head in order to get the robot in his field of view. Also, when the robot is far away from the human, the effect of the visibility must decrease. The computed visibility costs are shown in figure 3 . The zone situated in front of the human has very low costs. On the contrary, the zone situated behind the human has higher costs. Since the grid is attached to the head of the human, the computed costs are updated when the human changes his field of view (turn his head or his direction) during planning and/or execution stage.

\section{An extension: Hidden Zones}

In the grids illustrated above, the costs are calculated without taking into account the obstacles in the environment. However, obstacles in close vicinity of the human can have

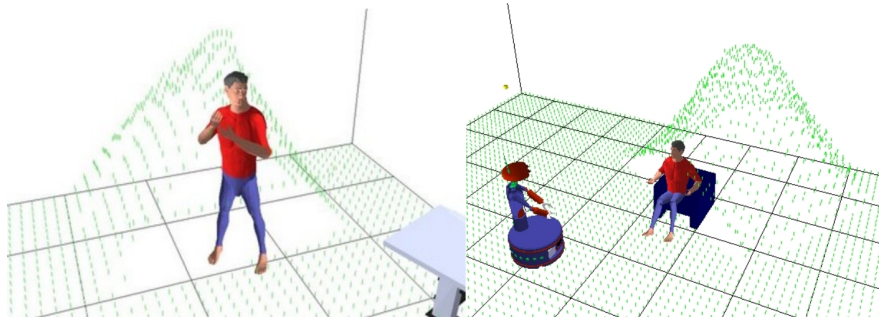

Fig. 3. Visibility grid is computed by taking into account human's field of view. Places that are difficult for the human to see have higher costs.

various effects on safety and comfort. If the robot is behind an obstacle, the human would feel secure because the obstacle blocks the direct path between the human and the robot. So the safety criterion must be canceled in the zones located behind the obstacles.

On the other hand, when the robot becomes hidden by an obstacle, the visibility costs lose their meanings. To handle this issue, we introduce an extension to visibility and safety, called "hidden zones" criterion. This criterion helps to determine better costs for positions hidden by the obstacles.

Another important effect of obstacles to human's comfort is the surprise factor. When the robot is hidden by an obstacle and suddenly appears in the human field of view, it can cause surprise and fear, especially if it is close to the human. To avoid this effect, we must discourage the robot from passing behind an obstacle too closely, and must constrain it to enter human's field of view sufficiently far away. This is done by putting costs to zones hidden by obstacles with respect to the human.

The costs in the hidden zone grid is inversely proportional to the distance between the human and the robot. In our system, the range of the surprise factor is approximately $3 \mathrm{~m}$, so the costs decreases to zero in the $3 \mathrm{~m}$ range and remains null for the other grid points (Fig. 4). These values can be additionally tuned according to the scenario and type of interaction.

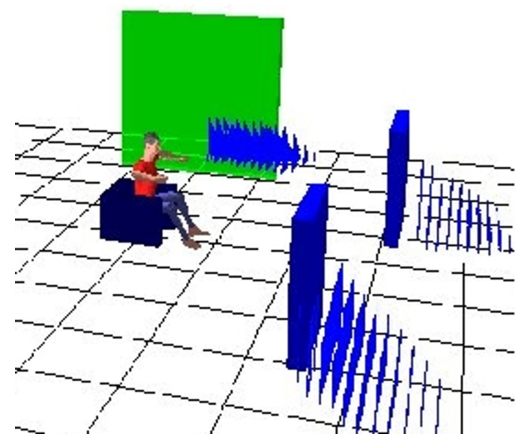

Fig. 4. Decreasing costs attributed to the zones hidden by obstacles. The supplementary costs discourage the robot getting too close to the obstacles and thus prevents the robot from appearing suddenly behind hidden places.

\section{Path planner}

Once the safety, visibility and hidden zones grids have been computed, they are merged into a single grid in which the robot 
will search for a minimum cost path. Different methods can be used to merge the grids. A first way can be to compute the overall cost from the weighted sum of the elementary costs:

$\operatorname{Cost}_{\text {merged }}(x, y)=w_{1} \operatorname{Cost}_{\text {safety }}(x, y)+w_{2} \operatorname{Cost}_{\text {visibility }}(x, y)$ where $(x, y)$ is a point in the grid, $w_{1}$ is the weight of the safety grid and $w_{2}$ is the weight of the visibility grid.

Another way is to consider the maximum cost values when merging the grids

$\operatorname{Cost}_{\text {merged }}(x, y)=\max \left(\operatorname{Cost}_{\text {safety }}(x, y), \operatorname{Cost}_{\text {visibility }}(x, y)\right)$

Note that we do not merge the hidden zones grid with the other two grids. That is mainly because the hidden zones grid serves as a replacement of these two grids for positions where the robot could not be seen because of an obstacle. The cost of a point $(x, y)$ in the final grid is computed by:

if $\left((x, y)\right.$ is in field of view of $\left.H_{i}\right) \wedge\left(H_{i}\right.$ cannot see $(x, y)$ because of obstacle $O)$ then

$$
\begin{aligned}
& \operatorname{Cost}_{\text {final }}(x, y) \leftarrow w_{3} \operatorname{Cost}_{\text {hiddenzones }}(x, y) \\
& \text { else } \\
& \quad \operatorname{Cost}_{\text {final }}(x, y) \leftarrow \operatorname{Cost}_{\text {merged }}(x, y) \\
& \text { end if }
\end{aligned}
$$

Our planner can use both merging methods depending on the task and on the balance between criteria. Also, the weights of the grids can be tuned according to the properties of the task.

To find a path between two given positions of the robot, we search for a path in the final grid that minimizes the sum of the costs of the cells linking the cells corresponding to these two positions. The cells corresponding to the obstacles in the environment are labeled as forbidden and an $A^{\star}$ search is performed to find a minimum-cost collision-free path linking two positions. The computed path is collision-free and also respects the human's safety and comfort by taking into account safety, visibility and hidden zones.

Neither the final grid nor 3 criterion grids are constructed explicitly but the values of the cells are calculated for the ones explored during $A^{\star}$ search. As humans in the environments can change their positions and orientations often, avoiding explicit grid construction gives us the possibility to replan a new path if a change in the environment occurs (i.e. change in human positions, orientations, or states).

\section{Simulation Results}

The Human Aware Motion Planner is implemented in $\mathrm{C}$ and integrated within the Move3D [22] software platform developed at LAAS.

Figure 5 illustrates two similar situations where two persons are in a conversation. The aim for the robot is to approach and join them. Although the robot can take the shortest path and pass between humans, the planner calculates a path longer but safer and more comfortable for both humans (Figure 5-a). By following this path, the robot does not approach too close to the humans when it is invisible, and enters the conversation in a more natural way by making a frontal approach. To illustrate the effect of obstacles in the environment, we place a wall in the same scenario, next to the human on the right (Figure 5b). Although the obstacle is not blocking the path of the robot and the path is still valid from a classical planning view, the robot calculates a new path. Because of the obstacle blocking a part of the human's field of view, the previous path becomes undesirable by making the robot suddenly appear too close. With this new path the robot enters smoothly into the view.

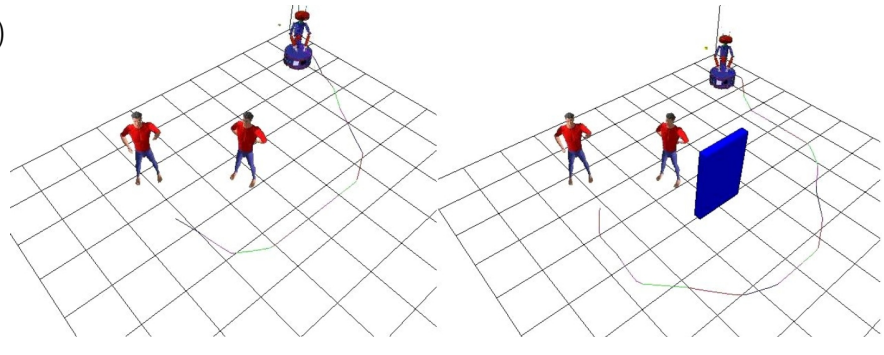

Fig. 5. A scenario with two persons talking and a robot that wants to join them. a) The planner calculates a path by taking into account safety and visibility. b) Although the previous path is valid, the planner finds a new path to avoid the surprise effect that can come from its sudden appearance in the humans' view.

The behavior of the Human Aware Motion Planner in a hallway is illustrated in figure 6 . In this scenario, the robot and a person cross in a hallway. The planner calculates a path to avoid a collision. Although the motion possibilities are restricted because of the environment, a friendly behavior appears. The robot avoids the human by moving to the right. After passing the human, instead of taking immediately its previous lane, the robot stays a certain distance from the human and thus behaves more friendly.

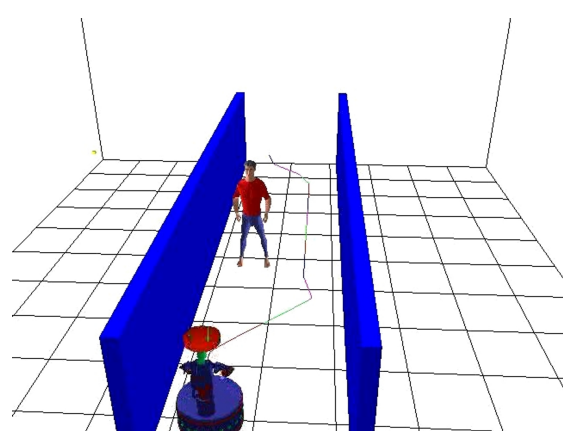

Fig. 6. A robot and a person cross in a hallway. The robot planner calculates a path that integrates a social behaviour: the robot avoids to come too close to the human's back.

Figure 7 illustrates another scenario with a person sitting in a room. The robot is initially located in the right corner of the room and has to move next to the human hidden by the wall obstacle. An example of the behaviour of a classical motion planner is shown in 7-a. Both paths are uncomfortable since the robot either passes too close and behind the human or appears suddenly in the human's field of view. 
The second figure (7-b) shows the path computed by the Human Aware Motion Planner. This path has the following characteristics:

- The robot does not approach too close to the humans. It chooses a solution that only enters in the humans $3 \mathrm{~m}$ zone in the last portion of the path.

- The robot remains as visible as possible along the path. Because of the hidden start position, there is no possibility to be in the human field of view at the beginning of path. Therefore the planner chooses to pass behind the wall instead of passing behind the human.

- The robot is not too close to the human when it appears in his field of view. The transition from the invisible zone behind the wall to the visible one is sufficiently far from the human to avoid any surprise effect. Then the robot can approach the human to reach its final position.

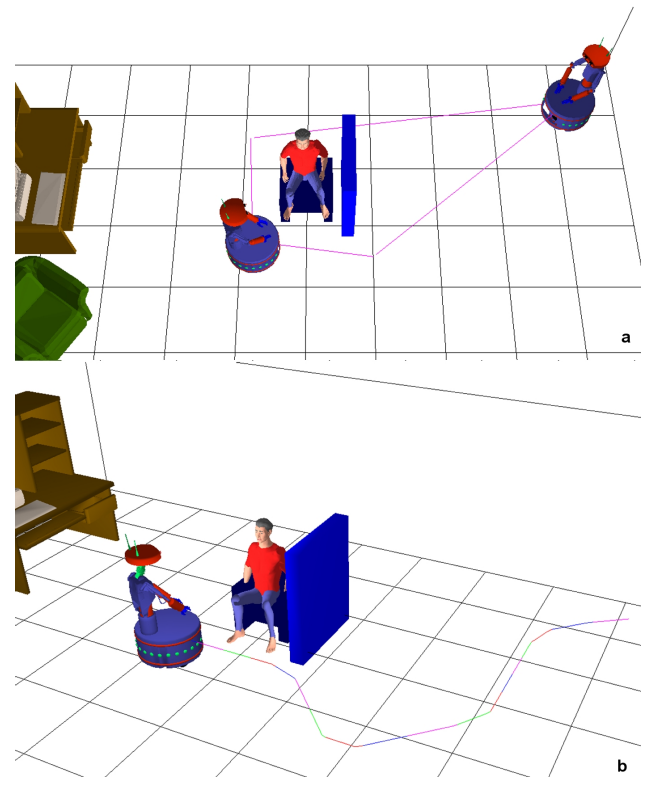

Fig. 7. A comparison between a standard motion planner and HAMP. Clearly paths produced by the first one are not acceptable since the robot either looms into the human's field of view or passes too near to the human's back. The path found by HAMP is more friendly with respect to safety, visibility, and hidden zones.

A last example of the features of our planner is illustrated in figure 8 representing an apartment scenario with two persons: Clark (with light shirt) and Bruce (with dark shirt). We look at the synthesized paths between the living room and the kitchen in different situations.

In figure 8-a, we show the path generated by the navigation planner for a situation in which Clark orders the robot to bring a sandwich from the kitchen. The computed motion takes into account the safety and the comfort of both humans by trying to stay in the visibility fields.

We can see in figure 8-b a computed path that avoids looming from behind the kitchen wall. Instead the robot chooses a path that keeps a certain distance to this wall.
TABLE I

COMPUTATION TIMES OF THE PATHS IN FIGURE 8

\begin{tabular}{|c||c||c||c||c|}
\hline Grid Resolution & Figure 8-a & Figure 8-b & Figure 8-c & Figure 8-d \\
\hline $0.2 \mathrm{~m}$ & 0.07 & 0.09 & 0.06 & 0.15 \\
\hline $0.1 \mathrm{~m}$ & 0.21 & 0.25 & 0.23 & 0.50 \\
\hline $0.05 \mathrm{~m}$ & 0.44 & 0.78 & 0.49 & 0.20 \\
\hline
\end{tabular}

In figure 8-c, we can see that Bruce came to talk to Clark, so the robot calculates a different path which stays in Clark's field of view and also avoids passing too near to Bruce's back.

The minimum cost approach of our navigation planner allows the robot to choose an alternative path if the path is blocked by an obstacle or a person as shown in figure 8-d where Bruce is blocking the passage.

Our planner is fast enough to replan and adapt its path along the execution. If a grid change occurs, like a change in human state, position, orientation or appearance of a an obstacle, fast computation times allow online replanning and a smooth switch to the new path. Table I shows the processing CPU-times on an AMD Athlon 1.8 GHz processor of the paths shown in figure 8 for 3 different grid resolutions.

\section{ROBOT IMPLEMENTATION \& RESULTS}

The planner is integrated into OpenGenom [23] as a module of the LAAS architecture [24]. As shown in the figure 9, the whole system has been carried to our robot Rackham, equipped with a front SICK laser scanner, a tilt \& pan camera, infrared proximity sensors and sonars with three Pentium III processors.

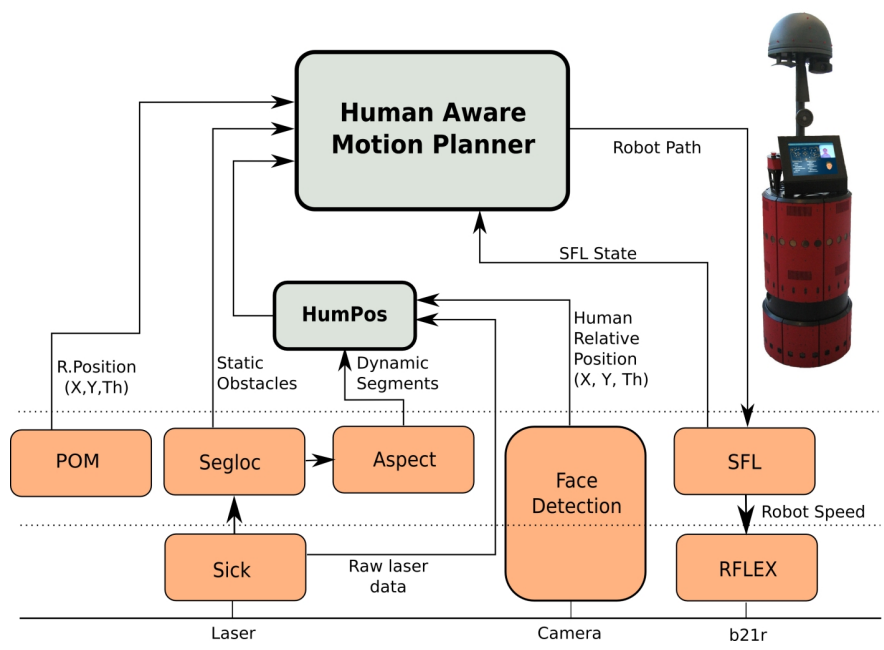

Fig. 9. General architecture of the robot composed of various OpenGenom [23] modules

Two additional modules are introduced into the system. As the positions and orientations of each human in the environment must be known, a human detection and tracking module (named humPos) has been developed. This module guarantees the data flow of human positions needed by the planner module. 


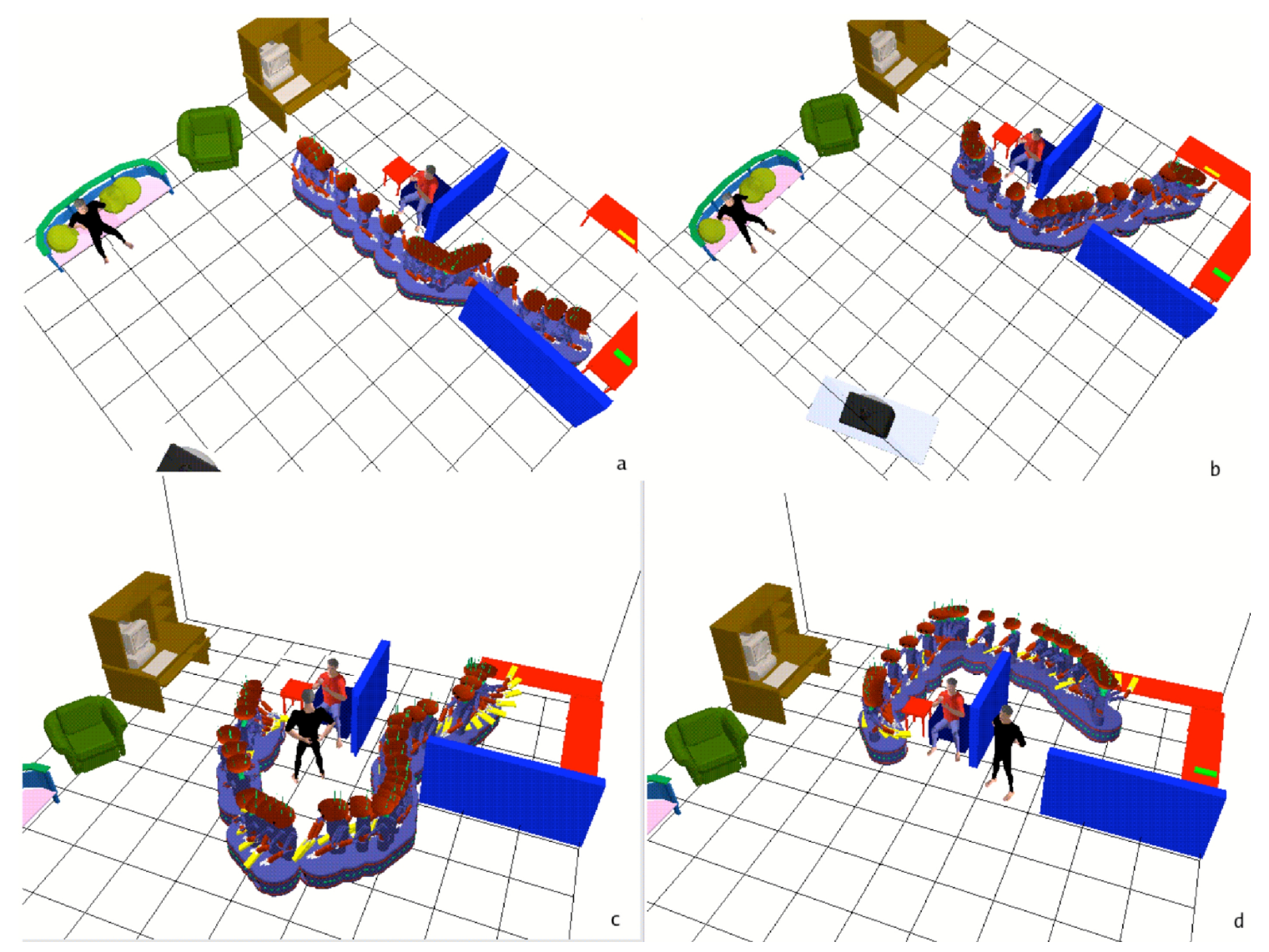

Fig. 8. Paths generated by the Human-Aware Motion Planner. Note that (1) the robot avoids suddenly appearing in the close vicinity of humans, (2) it tries to stay in their field of sight, and (3) it avoids approaching humans from behind.

\section{A. HumPos - Human Detection \& Tracking Module}

Detecting humans is necessary for a robotic/computer [25] system that involves interaction with humans. There are different methods depending on the robot's sensor capabilities. With camera and laser, the information can be used to detect more precisely humans in the robot's proximity [26]. In the absence of cameras, the laser can be used to detect leg-like shapes [27]. After the detection, tracking [28] [29] must be launched in order to follow the human motions and detect motion patterns.

For this purpose, we have developed the "HumPos" module, a module that provides human detection and tracking services based on laser and camera data. HumPos provides a list of humans in the environment to the motion planner. This list contains positions and orientations of the detected humans associated with a confidence index and an identifier.

The algorithm and methods used for laser based human detection and tracking are very simplistic and work under two assumptions:

- The gaze direction of a person is always the same as the direction of his body.

- A moving person is always moving forward looking at his motion direction.

The general algorithm consists of making two types of human detection (laser and visual), matching these two and tracking. At the end, an orientation assignment stage is per- formed on the results of the tracking. Figure 10 shows the overall mechanism of human detection and tracking.

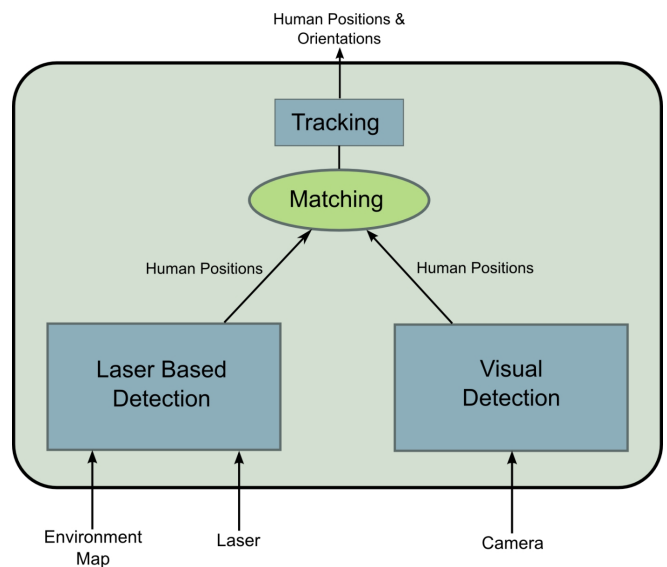

Fig. 10. The Human Detection process combines laser and visual data to detect and track humans.

In laser based detection, static obstacles in the environment map are filtered from the sensor data. Resulting points are then used to detect leg-like shapes (a leg or pair of legs) according to their geometry and neighborhood. This process produces a list of detected humans with their positions and an attached confidence index. 
On the other hand, the visual data coming from the camera are used to detect people in near proximity of the robot by the visual face detection module (figure 11-b). The visual face detection module provides a list of humans looking directly at the robot with their estimated distance based on facial size metrics within a range of approx. 1 to 3 meters [30].

These two lists are then matched to produce only one list of humans with corresponding positions, orientations, and confidence index (figure 11-c). Finally, detected humans are tracked by the tracking stage. At the end of this stage, orientations are assigned to detected humans according to their motions, the visual detection result and, the 2 assumptions that we made above. The orientation assignment procedure is described in Algorithm 1.

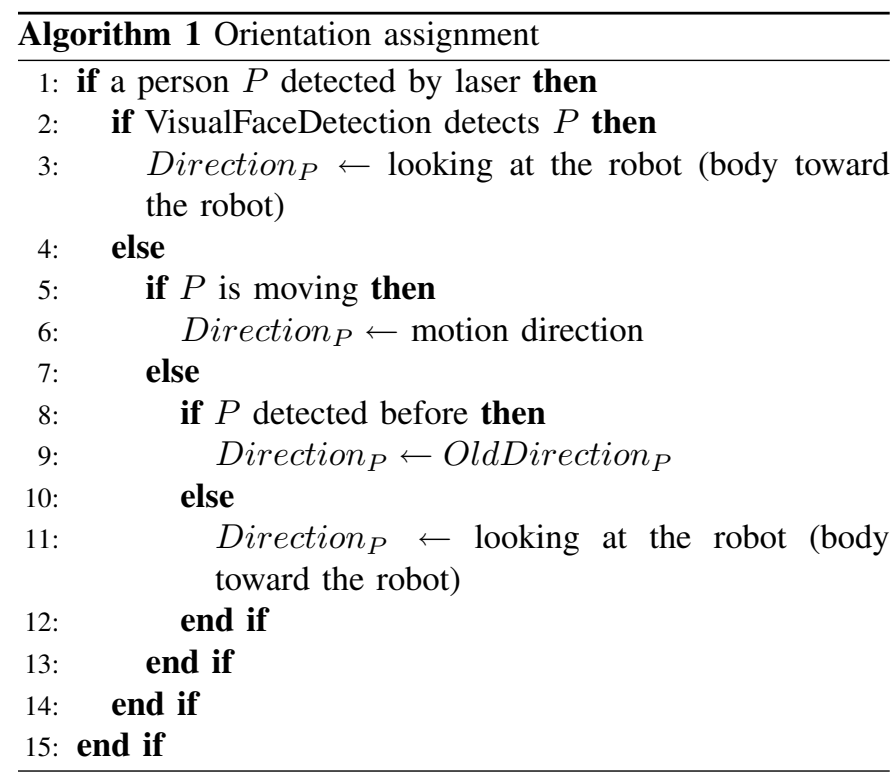

If a person is looking at the robot and thus detected visually, we assign his orientation to the direction of the robot. If the visual face detection fails, then laser based leg detection decides humans' orientations. If a person is detected and he is moving, his motion direction is assigned as his head/body orientation. If a person stops, his last orientation is conserved and assigned to next detections until he moves, disappears, or is detected by visual face detection.

\section{B. Planner Module}

The planner module works with a static internal 3D map along with each human's model, his grid construction parameters and the robot model. The humans' positions are updated by the HumPos module and the robot's current position is updated by Position Manager module. A constant data flow from HumPos to the planner is necessary to maintain information about humans. With these inputs (figure 12), the planner module calculates a path that takes into account the safety and visibility constraints explained in Section III.

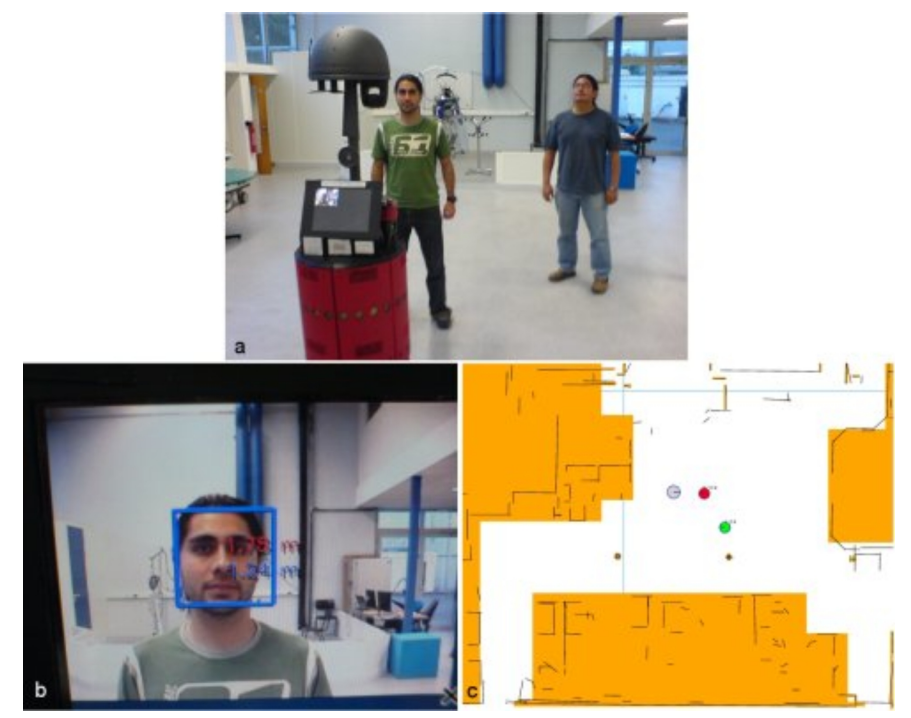

Fig. 11. a- Two persons have been detected based on laser data b- One of them is also detected using a vision-based face detection c- The one detected by the camera has high probability and is marked with red while the other person is marked with a lower probability.

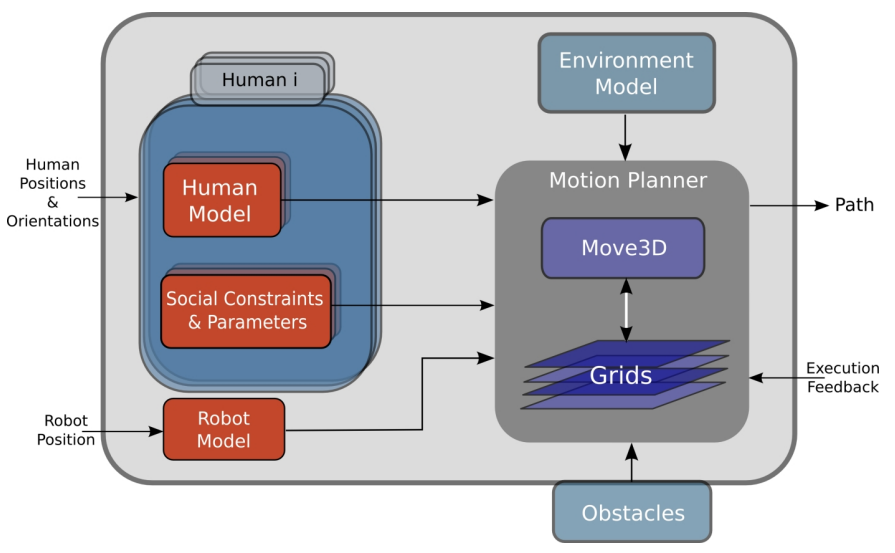

Fig. 12. Architecture of the Human Aware Motion Planner module

The computed path is then sent to the execution module [31]. This module produces a smooth trajectory following the path given in the form of a set of passage points. If the system detects a new person or a change of position, orientation, or state of an existing person during the robot's motion, the planner replans a new path and sends it to the execution module. Since the robot is in motion, the planner replans a new path that begins with a small portion of the old path. With this property the robot passes smoothly from the current path to a new one. To avoid constant replanning and possible errors from the human detection phase, the planner only replans a new path if a human position changes 0.2 meters or his orientation changes 0.3 radians.

\section{Implementation Results}

In figure 13, a scenario with two people in a conversation and a comparison between a standard motion planner and 


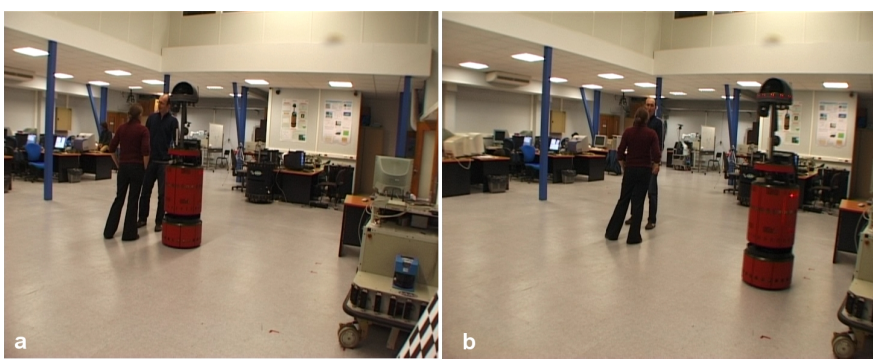

Fig. 13. A comparison between a classic motion planner and the Human Aware Motion Planner which produces a more acceptable path by taking into account the safety and visibility of each human in the environment.

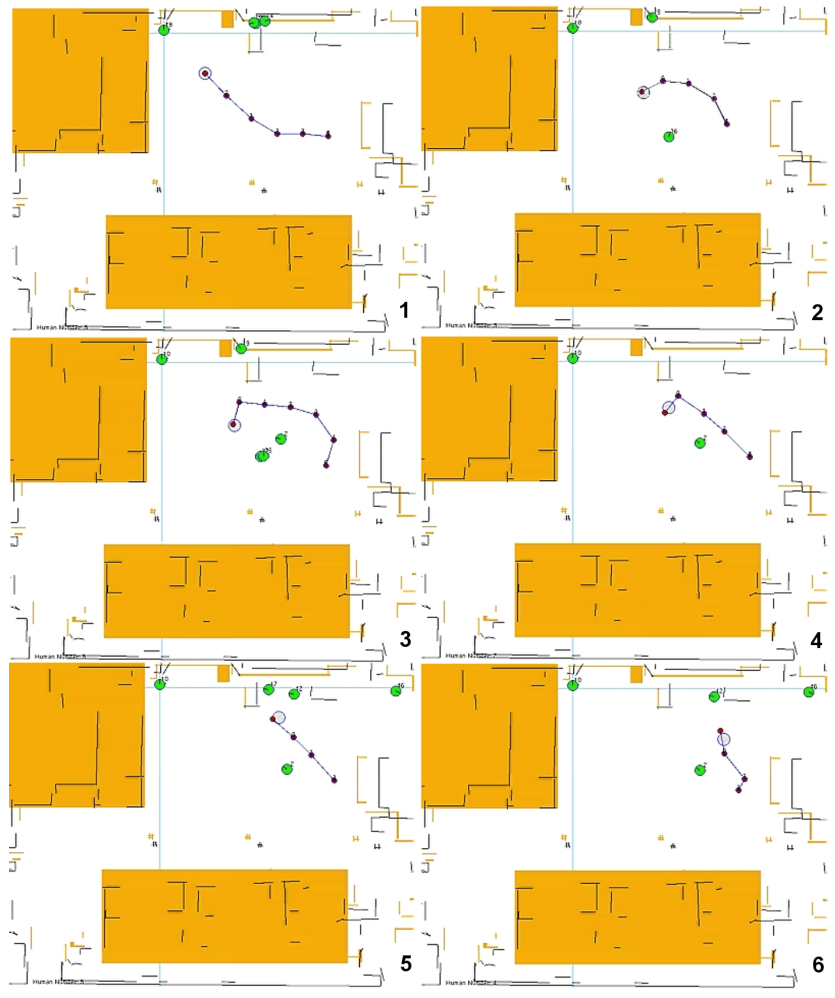

Fig. 14. Replanned path during the execution of a trajectory. The path is then recalculated in case of a change in the environment and executed immediately.

Human Aware Motion Planner are illustrated. In this scenario, the robot aims to move from one corner of the room to the other. The direct path between these two points is blocked by two people. One of the humans has his back turned to the robot and thus can not see the robot.

Using a "classical" motion planner, the robot tries to follow the shortest path to get to its goal. The humans are considered as obstacles. When the robot approaches sufficiently close to them, it modifies its path just enough to avoid them (figure 13-a). When the robot passes next to them, it causes surprise to the one who has not see the robot coming. Then it reaches the goal with a direct path.

Then, we replace the planner by HAMP. As can be seen in figure $13-b$, the robot does not approach directly to the humans because it cannot be seen by one of them. Thus it takes a larger distance to avoid any surprise and fear and it enters more smoothly into their field of view.
The initial path and replanned paths can be seen in figure 14. As the robot does not detect any one, it calculates a direct path. During its motion, it detects humans on its path and replans to adapt to their presence.

Figure 15 shows the effect of the visibility criterion. Following visibility costs, a path costs less when the robot is in front of the human than a path situated behind. That is why Rackham "prefers" to pass in front of the human in figure 15a. However in situations where there is not enough free space in front of the human (or blocked by an obstacle or another human), the robot passes behind, giving a larger distance to the human (figure 15-b).

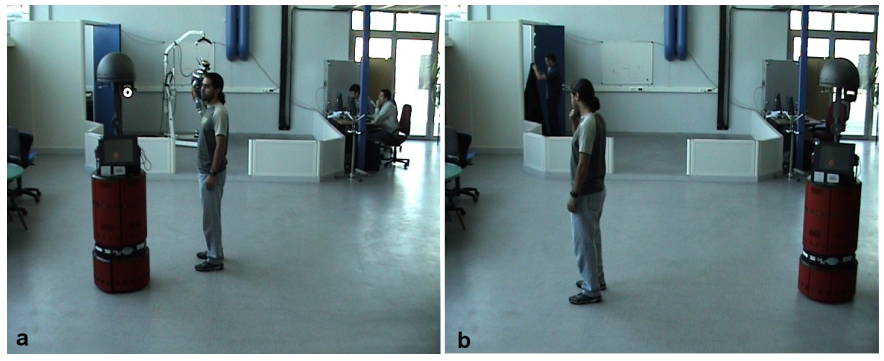

Fig. 15. Visibility criterion effect when passing in front or behind of a human.

Another scenario is illustrated in figure 16 where a person and robot move toward each other. The robot follows a straight path before detecting the human (figure 16-a). Once the human is detected, the replanning produces a new path to avoid him (figure 16-b). After passing the human, the robot doesn't immediately take its previous lane but it gives a little distance to the human's back (figure 16-c). This behavior avoids possible unpleasantness in case of a change in the human's motion.

The final example shows the effect of hidden zones (Section III). In this scenario (figure 17), a whiteboard separates the human and the robot. Because of this obstacle, the robot is completely hidden from the human. On the robot's side, the human is partially hidden because his legs are visible to robot's laser. The robot detects that there is a person behind the whiteboard. Since the human approached the whiteboard before, his correct orientation coming from the leg tracking is kept by the robot. So the robot plans a path that takes into account not only safety and visibility but also the surprise effect coming from looming into the human's field of view. By following this path, the robot gets farther from the whiteboard. Then once it is visible, it advances toward its goal normally.

Further examples and complete videos can be found at http://www.laas.fr/ easisbot. Although the planner produces different paths depending on human state such as the posture (see Figures 2 and 8), this feature has not been tested on the real experiments since the perception system we use is not able to provide such information. 


\section{CONCLUSION AND FUtURE Work}

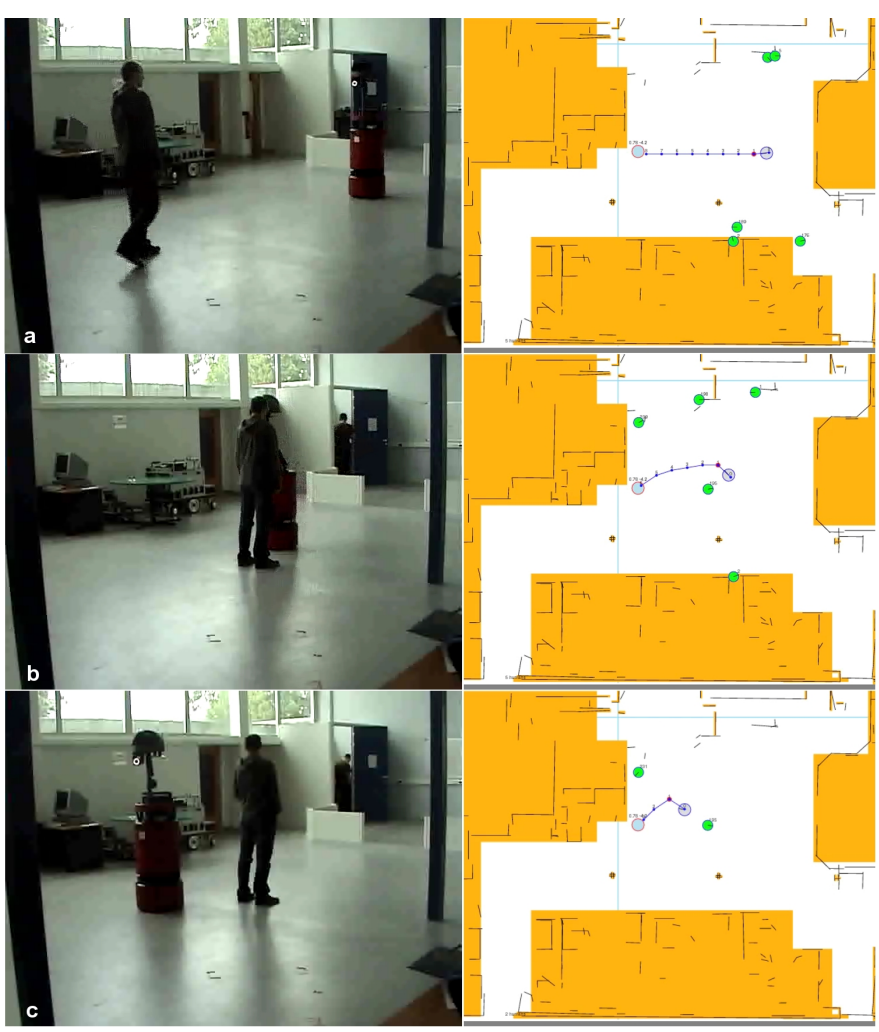

Fig. 16. A person and robot move toward each other. The robot changes it's trajectory to avoid a collision. After passing next to the human instead of taking immediately its previous lane, it gives a little distance to the human. This behavior ensures a comfortable and riskless motion for the human.

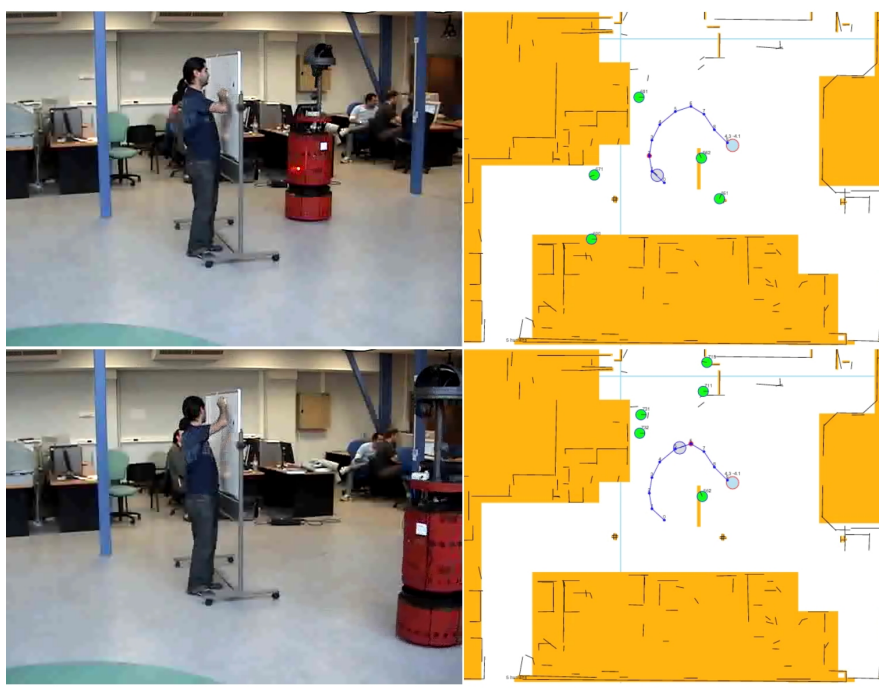

Fig. 17. Robot is invisible to the human. To avoid any unpleasantness coming from the sudden appearance of the robot, the planner calculates a path which make the robot appear farther.
We have presented algorithms of a motion planner reasoning on humans' positions, fields of view, and postures. Our planner produces robot paths significantly different from those produced by "standard" motion planners. Although the planner takes the humans as static, not moving, entities, fast processing times allow replanning on-line to assure a good reactivity in case of moving humans.

We integrated the planner in our robotic platform Rackham. We presented a supplementary human detection module sufficiently accurate for our needs. The planner is run on different scenarios and the results showed the feasibility of our approach.

The robot dynamics along its trajectory is also a very important aspect to be taken into account in human-robot interaction scenario. It can have a major effect on the comfort and safety of the humans. One of the next steps will be the adaptation of robot speed and acceleration to produce more friendly motions. In one of our previous works [20], we have proposed a planner that deals implicitly with this issue by adapting the robot's speed to avoid a collision in case of a possible sudden appearance of a human. This work can be merged with human aware motion planner and can be very beneficial for both of the systems. This consideration will allow us to improve the human awareness of our planner by not only reasoning on spacial coordinates but also on the whole robot motion including speed and time considerations.

Although the human detection module provides an important input to the planner, this module is developed to evaluate our planner. The detection of humans' positions and orientations provides sufficiently correct data in experiment environments under some assumptions, but clearly it is not enough to be carried to a real world home scenario. A more powerful and robust human position and especially orientation detection will certainly improve the quality and correctness of the paths produced by the planner. This is certainly a key issue that is developed by our team and other research groups but is not in the scope of this paper.

Other future work will be on validating our navigation planner. Previous user studies have permitted us to exhibit some key issues, now further user studies have to be conducted in order to evaluate the effectiveness of the whole system.

Even though our human-aware motion planner provides already socially acceptable motion of the robot when it approaches or "encounters" a person, there is still much to do and a number of questions are still open. For instance, depending on the situation and the task at hand the robot might decide to move or simply to stay still, or to yield the way because it is blocking a passage. Other behaviours such as guiding, accompanying or following are task-dependent and will consequently influence the motion planner choices. These questions clearly enlarge the problem.

Following this path, we are currently investigating how to extend our approach in order to produce behaviours for handing an object to a person. Indeed, there is a need to take into account visibility and reachability, in terms of kinematic constraints, of the human partner. Besides, the robot should 
produce motion that is acceptable and easily "legible". The human partner should easily understand by observing the robot motion that its intention is to hand an object.

One additional difficulty when considering such issues is the construction of a coherent framework that allows to take into account various constraints of different nature. For instance, some of them can be best expressed geometrically while others may be expressed in terms of temporal synchronization or task-dependent social conventions. By taking motion and manipulation planner as a whole, the system can be extended to produce paths and tasks not only for the robot but also for its human partner. Such a system will reason about human motions, tasks and coordination events in relation with robot motion execution.

\section{ACKNOWLEDGMENT}

The work described in this paper was partially conducted within the EU Integrated Project COGNIRON [32] ("The Cognitive Companion") and funded by the European Commission Division FP6-IST Future and Emerging Technologies under Contract FP6-002020.

The authors want to thank the anonymous reviewers for their very constructive feedback that helped us to strengthen the paper.

\section{REFERENCES}

[1] T. Fong, I. Nourbakhsh, and K. Dautenhahn, "A survey of socially interactive robots," Robotics and Autonomous Systems, vol. 42, pp. 143166, 2003.

[2] R. Alami, R. Chatila, A. Clodic, S. Fleury, M. Herrb, V. Montreuil, and E. A. Sisbot, "Towards human-aware cognitive robots," The Fifth International Cognitive Robotics Workshop (The AAAI-06 Workshop on Cognitive Robotics), 2006.

[3] E. A. Sisbot, R. Alami, T. Siméon, K. Dautenhahn, M. L. Walters, S. N. Woods, K. L. Koay, and C. L. Nehaniv, "Navigation in the presence of humans," Proc. IEEE-RAS International Conference on Humanoid Robots, pp. 181-188, 2005.

[4] E. A. Sisbot, L. F. M. Urias, R. Alami, and T. Siméon, "A mobile robot that performs human acceptable motion," Proc in (IEEE/RSJ) International Conference on Intelligent Robots and Systems, 2006.

[5] K. Dautenhahn, M. Walters, S. Woods, K. L. Koay, C. Nehaniv, E. Sisbot, R. Alami, and T. Simeon, "How may i serve you?, a robot companion approaching a seated person in a helping context," Conference on Human-Robot Interaction, 2006.

[6] R. Alami, A. Albu-Schaeffer, A. Bicchi, R. Bischoff, R. Chatila, A. D. Luca, A. D. Santis, G. Giralt, J. Guiochet, G. Hirzinger, F. Ingrand, V. Lippiello, R. Mattone, D. Powell, S. Sen, B. Siciliano, G. Tonietti, and L. Villani, "Safe and dependable physical human-robot interaction in anthropic domains: State of the art and challenges," in Proc. IROS'06 Workshop on pHRI - Physical Human-Robot Interaction in Anthropic Domains, A. Bicchi and A. D. Luca, Eds. IEEE, 2006.

[7] S. Nonaka, K. Inoue, T. Arai, and Y. Mae, "Evaluation of human sense of security for coexisting robots using virtual realit, 1st report," in Proc. (IEEE) International Conference on Robotics \& Automation, New Orleans, USA, 2004.

[8] A. Bicchi and G. Tonietti, "Fast and soft arm tactics: Dealing with the safety-performance trade-off in robot arms design and control," Robotics and Automation Magazine, vol. 11, no. 2, pp. 22-33, 2004.

[9] M. Zinn, O. Khatib, B. Roth, and J. K. Salisbury, "Playing it safe [human-friendly robots]," Robotics \& Automation Magazine, vol. 11, no. 2, pp. 12-21, 2004.

[10] B. Lussier, A. Lampe, R. Chatila, J. Guiochet, F. Ingrand, M. O. Killijian, and D. Powell, "Fault tolerance in autonomous systems: how and how much?" 4th IARP/IEEE-RAS/EURON Workshop on Technical Challenges for Dependable Robots in Human Environments, 2005.

[11] D. Kulic and E. A. Croft, "Real-time safety for human robot interaction," Proc. of the International Conference on Advanced Robotics, 2005.
[12] M. Nokata, K. Ikuta, and H. Ishii, "Safety-optimizing moethod of human-care robot design and control," Proc. in (IEEE) International Conference on Robotics \& Automation, pp. 1991-1996, 2002.

[13] K. Ikuta, H. Ishii, and M. Nokata, "Safety evaluation methods of design and control for human-care robots," The International Journal of Robotics Research, vol. 22, no. 5, pp. 281-297, 2003.

[14] R. S. Rao, K. Conn, S. H. Jung, J. Katupitiya, T. Kientz, V. Kumar, J. Ostrowski, S. Patel, and C. J. Taylor, "Human-robot interaction: Application to smart wheelchairs," Proc. in (IEEE) International Conference on Robotics \& Automation, 2002.

[15] E. T. Hall, The Hidden Dimension. Garden City, N.Y.: Doubleday, 1966.

[16] R. Chatila, R. Alami, T. Simeon, J. Pettre, and L. Jaillet, "Safe, reliable and friendly interaction between humans and humanoids," 3rd IARP International Workshop on Humanoid and Human Friendly Robotics, pp. 83-87, 2002.

[17] P. Althaus, H. Ishiguro, T. Kanda, T. Miyashita, and H. I. Christensen, "Navigation for human-robot interaction tasks," Proc. in IEEE Int. Conf. on Robotics \& Automation, New Orleans, USA, 2004.

[18] E. Pacchierotti, P. Jensfelt, and H. Christensen, "Design of an officeguide robot for social interaction studies," Proc. in (IEEE/RSJ) International Conference on Intelligent Robots and Systems, pp. 4965-4970, 2006.

[19] E. Pacchierotti, H. Christensen, and P. Jensfelt, "Embodied social interaction for service robots in hallway environments," Field and Service Robotics, pp. 476-487, 2005.

[20] K. M. Krishna, R. Alami, and T. Simeon, "Safe proactive plans and their execution," Robotics and Autonomous Systems, 2005.

[21] M. Walters, K. Dautenhahn, R. te Boekhorst, K. L. Koay, C. Kaouri, S. Woods, C. Nehaniv, D. Lee, and I. Werry, "The influence of subjects personality traits on personal spatial zones in a human-robot interaction experiment," IEEE International Symposium on Robot and Human Interactive Communication, 2005.

[22] T. Siméon, J.-P. Laumond, and F. Lamiraux, "Move3d: a generic platform for motion planning," in 4th International Symposium on Assembly and Task Planning, Japan, 2001.

[23] S. Fleury, M. Herrb, and R. Chatila, "Genom: A tool for the specification and the implementation of operating modules in a distributed robot architecture," in In International Conference on Intelligent Robots and Systems, Grenoble, France, 1997, pp. 842-848.

[24] R. Alami, R. Chatila, S. Fleury, M. Ghallab, and F. Ingrand, "An architecture for autonomy," International Journal of Robotic Research, vol. 17, pp. 315-337, 1998.

[25] Computers in the human interaction loop - chil website. [Online]. Available: http://chil.server.de/servlet/is/101/

[26] M. Kleinehagenbrock, S. Lang, J. Fritsch, F. Lomker, G. A. Fink, and G. Sagerer, "Person tracking with a mobile robot based onmultimodal anchoring," in Int. Workshop on Robot and Human Interactive Communication, Berlin, Germany, 2002.

[27] J. Xavier, M. Pacheco, D. Castro, and A. Ruano, "Last line, arc/circle and leg detection from laser scan data in a player driver," in In IEEE International Conference on Robotics and Automation, Barcelona, Spain, 2005.

[28] D. Shulz, W. Burgard, D. Fox, and A. Cremers, "Tracking multimple moving objects with a mobile robot," in Proc. of the IEEE Computer Society Conference on computer vision and pattern recognition (CVPR), Kauai,HW, 2001.

[29] A. Baba and R. Chatila, "Simultaneous environment mapping and mobile target tracking," International Conference on Intelligent Autonomous Systems, 2006.

[30] L. Brèthes, F. Lerasle, and P. Danès, "Data fusion for visual tracking dedicated to human-robot interaction," International Conference on Robotics and Automation, pp. 2087-2092, 2005.

[31] R. Philippsen, "Motion planning and obstacle avoidance for mobile robots in highly cluttered dynamic environments," Ph.D. dissertation, Ecole Polytechnique Fédérale de Lausanne, 2004.

[32] The cognitive robot companion - cogniron website. [Online]. Available: http://www.cogniron.org/ 